\title{
Spanning paths in hypercubes
}

\author{
Tomáš Dvořák ${ }^{1}$, Petr Gregor ${ }^{1}$ and Václav Koubek ${ }^{1,2, \dagger}$ \\ ${ }^{1}$ Faculty of Mathematics and Physics, Charles University, Malostranské nám. 25, 11800 Praha, Czech Republic \\ ${ }^{2}$ Institute for Theoretical Computer Science, Charles University, Malostranské nám. 25, 11800 Praha, Czech Repub- \\ lic
}

Given a family $\left\{u_{i}, v_{i}\right\}_{i=1}^{k}$ of pairwise distinct vertices of the $n$-dimensional hypercube $Q_{n}$ such that the distance of $u_{i}$ and $v_{i}$ is odd and $k \leq n-1$, there exists a family $\left\{P_{i}\right\}_{i=1}^{k}$ of paths such that $u_{i}$ and $v_{i}$ are the endvertices of $P_{i}$ and $\left\{V\left(P_{i}\right)\right\}_{i=1}^{k}$ partitions $V\left(Q_{n}\right)$. This holds for any $n \geq 2$ with one exception in the case when $n=k+1=4$. On the other hand, for any $n \geq 3$ there exist $n$ pairs of vertices satisfying the above condition for which such a family of spanning paths does not exist. We suggest further generalization of this result and explore a relationship to the problem of hamiltonicity of hypercubes with faulty vertices.

Keywords: Hamiltonian paths, spanning paths, hypercube, vertex fault tolerance

\section{Introduction}

The $n$-dimensional hypercube $Q_{n}$ is an undirected graph whose vertex set consists of all binary vectors of length $n$, two vertices being joined by an edge whenever their binary representations differ in a single coordinate. The structural properties of hypercubes have been a subject of interest to graph theoreticians (cf. a survey [8]) as well as to computer scientists due to the application of hypercubes as prospective interconnection networks for parallel or distributed computation (cf. a monograph [11]).

The existence of a hamiltonian cycle (path) in $Q_{n}$ for any $n \geq 2(n \geq 1)$ is a well-known and a widely studied fact (cf. [13]). Note that as any hypercube is a bipartite graph with an even number of vertices, endvertices of any hamiltonian path must belong to different partite sets. A classical result of Havel ([9]) says that this obvious necessary condition is also sufficient, i. e. for any $n \geq 1, Q_{n}$ contains a hamiltonian path between a given pair of vertices $u, v$ iff $u$ and $v$ belong to different partite sets. A further generalization was recently obtained by Caha and Koubek as one of results in [2]: Given a family $\left\{u_{i}, v_{i}\right\}_{i=1}^{k}$ of pairwise distinct vertices of $Q_{n}$ such that $u_{i}$ and $v_{i}$ belong to different partite sets and $k \leq(n-1) / 3$, there exists a family of paths $\left\{P_{i}\right\}_{i=1}^{k}$ such that $u_{i}, v_{i}$ are endvertices of $P_{i}$ and $\left\{V\left(P_{i}\right)\right\}_{i=1}^{k}$ partitions $V\left(Q_{n}\right)$. They also asked about the maximum value of $k$ for which such spanning paths exist. Our main result in Section 3 resolves this problem.

The applications of hypercubic networks in parallel computing stimulated the research of hamiltonicity of hypercubes with faulty edges and vertices. The problem may be formulated as follows: If certain edges or vertices of $Q_{n}$ are removed, does the remaining graph still contain a hamiltonian cycle or path?

\footnotetext{
${ }^{\dagger}$ Supported by the grant No. 1ET100300517 of the Czech Information Society.

1365-8050 (c) 2005 Discrete Mathematics and Theoretical Computer Science (DMTCS), Nancy, France
} 
While the variant with faulty edges was settled by results of Chan and Lee (in [3] they prove that $Q_{n}$ remains hamiltonian even after the removal of at most $2 n-5$ edges provided that the resulting graph has minimum degree at least two) and of Tsai ([14] extends the previous result to hamiltonian paths with given endvertices), the status of the problem with faulty vertices is less satisfactory. Lewinter and Widulski proved in [12] that for any distinct vertices $u, v$ and $w$ of $Q_{n}, Q_{n}-\{w\}$ contains a hamiltonian path between $u$ and $v$ iff $u, v$ belong to the same partite set, different from that containing $w$. In Section 4 we formulate a conjecture generalizing this result to the case with a nonconstant number of faulty vertices. The other results we are aware of only say that if $f$ vertices are removed from $Q_{n}$, the resulting graph contains a cycle of length at least $\left|V\left(Q_{n}\right)\right|-2 f$ (Fu in [6] proves this for $f \leq 2 n-4$ ).

\section{Concepts and notation}

Our terminology mostly follows [7]. The vertex and edge sets of a graph $G$ are denoted by $V(G)$ and $E(G)$, respectively. The distance of vertices $u, v \in V(G)$ is denoted by $d(u, v)$. Given a set $V^{\prime} \subseteq V(G)$, $G-V^{\prime}$ denotes the subgraph of $G$, induced by the vertices of $V \backslash V^{\prime}$. A family $\left\{P_{i}\right\}_{i=1}^{k}$ of paths (cycles) of $G$ forms a family of spanning paths (spanning cycles) of $G$ if $\left\{V\left(P_{i}\right)\right\}_{i=1}^{k}$ partitions $V(G)$.

The norm of a vertex $v \in V\left(Q_{n}\right)$ is defined as the number of ones in the binary representation of $v$. The dimension of an edge $\{u, v\} \in E\left(Q_{n}\right)$ is the integer $i$ such that $u$ and $v$ differ in the $i$-th coordinate. Edges of the same dimension are called parallel. Let $\mathcal{F}$ be a family of pairs $\left\{u_{i}, v_{i}\right\}_{i=1}^{k}$ of vertices of $Q_{n}$ such that $\left\{u_{i}, v_{i}\right\} \cap\left\{u_{j}, v_{j}\right\}=\emptyset$ for all $i \neq j \in\{1,2, \ldots, k\}$. We call $\mathcal{F}$

connectable if there exists a family $\left\{P_{i}\right\}_{i=1}^{k}$ of spanning paths of $Q_{n}$ such that $P_{i}$ is a path between $u_{i}$ and $v_{i}$ for every $i=1,2, \ldots, k$;

odd if $d\left(u_{i}, v_{i}\right)$ is odd for all $i=1,2, \ldots, k$;

balanced if the number of pairs with both vertices of even norm equals the number of pairs with both vertices of odd norm.

Note that the definition of a balanced family allows that $u_{i}=v_{i}$ for some $i$, and therefore e.g.

$$
\{\{(00),(00)\},\{(01),(10)\}\}
$$

is a balanced family of vertices of $Q_{2}$.

\section{Results}

The following result was proved by Caha and Koubek in [2]:

Theorem 3.1 ([2]) Let $n \geq 2$ and $\left\{u_{i}, v_{i}\right\}_{i=1}^{k}$ be an odd family of vertices of $Q_{n}$. If $k \leq(n-1) / 3$, then $\left\{u_{i}, v_{i}\right\}_{i=1}^{k}$ is connectable.

Our main result improves the upper bound on the size of the connectable family.

Theorem 3.2 Let $n \geq 2$ and $\left\{u_{i}, v_{i}\right\}_{i=1}^{k}$ be an odd family of vertices of $Q_{n}$. If $k \leq n-1$, then $\left\{u_{i}, v_{i}\right\}_{i=1}^{k}$ is connectable unless $n=4, k=3, d\left(u_{i}, v_{i}\right)=3$ for all $i \in\{1,2,3\}$ and the subgraph induced by $\left\{u_{i}, v_{i}\right\}_{i=1}^{3}$ forms a cycle of length 6 . 


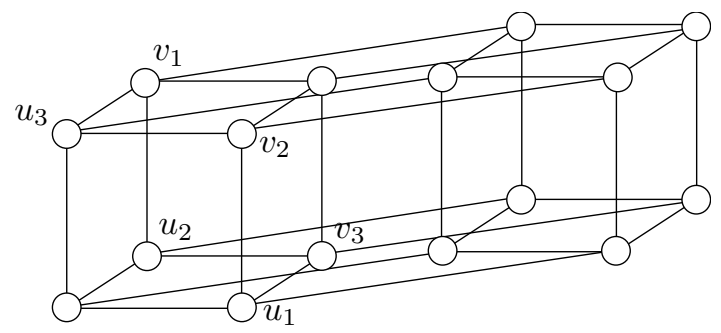

Fig. 1: The only non-connectable odd family of three pairs of vertices of $Q_{4}$

The only exceptional configuration in the case when $n=k+1=4$ is depicted on Figure 1 .

It is not difficult to see that the upper bound on the size of the family of vertex pairs for which Theorem 3.2 holds is actually sharp.

Proposition 3.3 For any $n \geq 3$ there exists an odd family of $n$ pairs of vertices of $Q_{n}$ which is not connectable.

Indeed, let $u_{1}, \ldots, u_{n}$ be distinct neighbors of an arbitrary vertex $u \in V\left(Q_{n}\right)$. Observe that as $n \geq 3$, we can choose pairwise distinct vertices $v_{1}, \ldots, v_{n}$ such that for any $i \in\{1,2, \ldots, n\}, v_{i}$ is a neighbor of $u_{i}$ distinct from $u$. Now if there is a path between $u_{i}$ and $v_{i}$ for some $i \in\{1,2, \ldots, n\}$ passing through $u$, it must also contain $u_{j}$ for some $j \neq i$. Hence $\left\{u_{i}, v_{i}\right\}_{i=1}^{n}$ forms an odd family of vertices of $Q_{n}$ which is not connectable.

If the family of spanning paths which exists by Theorem 3.2 contains no path of length one and $d\left(u_{i}, v_{i}\right)=1$ for all $i \in\{1,2, \ldots, k\}$, then we actually have a family of spanning cycles $\left\{C_{i}\right\}_{i=1}^{k}$ passing through given edges $\left\{u_{i}, v_{i}\right\} \in E\left(C_{i}\right)$. It should be noted that spanning cycles of hypercubes were previously studied by Kobeissi and Mollard in [10], where they construct spanning cycles of given lengths, each passing through a prescribed edge and avoiding a set of forbidden edges, provided all prescribed and forbidden edges are parallel. Hamiltonian cycles and paths of hypercubes passing through a given set of prescribes edges of arbitrary dimensions were studied in $[1,4,5]$.

\section{Conjectures}

The following result was proved by Caha and Koubek in [2]:

Theorem 4.1 ([2]) Let $n \geq 2$ and $\left\{u_{i}, v_{i}\right\}_{i=1}^{k}$ be a family of pairwise distinct vertices of $Q_{n}$. If $k \leq$ $(n+1) / 3$, then $\left\{u_{i}, v_{i}\right\}_{i=1}^{k}$ is connectable iff it is balanced.

We conjecture that this result may be improved by increasing the upper bound on the number of vertex pairs and weakening the requirement that each pair must consist of distinct vertices.

Conjecture 4.2 Let $n \geq 2$ and $\left\{u_{i}, v_{i}\right\}_{i=1}^{k}$ be a balanced family of vertices of $Q_{n}$ such that $u_{i} \neq v_{i}$ for some $i \in\{1,2, \ldots, k\}$. If $k \leq n-1$, then $\left\{u_{i}, v_{i}\right\}_{i=1}^{k}$ is connectable unless

(i) $n=k+1=3, d\left(u_{1}, v_{1}\right)=d\left(u_{2}, v_{2}\right)=2$ and $\left\{u_{1}, u_{2}\right\},\left\{v_{1}, v_{2}\right\}$ are parallel edges, or

(ii) $n=k+1=4, d\left(u_{i}, v_{i}\right)=3$ for all $i \in\{1,2,3\}$ and the subgraph induced by $\left\{u_{i}, v_{i}\right\}_{i=1}^{3}$ forms $a$ cycle of length 6 . 
So far we have been able to verify the validity of the conjecture for $n \leq 5$ by a computer search. The only two (up to isomorphism) exceptional configurations in the case when $n=3$ are depicted on Figure 2.
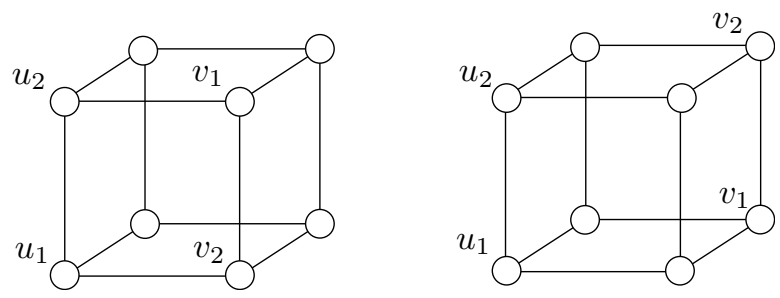

Fig. 2: The only non-connectable balanced families of two pairs of vertices of $Q_{3}$

Note that if true, then the upper bound $n-1$ is certainly the best possible, as demonstrated by Proposition 3.3. Moreover, the validity of the conjecture would also partially resolve the problem of hamiltonicity of $Q_{n}$ with faulty vertices. Indeed, if $u_{1} \neq v_{1}$ and $u_{i}=v_{i}$ for all $i \in\{2, \ldots k\}$, then Conjecture 4.2 simply says that $Q_{n}-\left\{u_{2}, \ldots, u_{k}\right\}$ contains a hamiltonian path between $u_{1}$ and $v_{1}$ iff the family $\left\{u_{i}, v_{i}\right\}_{i=1}^{k}$ is balanced. This is known to be true for $k \leq 2$ by the results of [9] and [12], quoted in Section 1.

We conclude this discussion with a question related to the complexity of our problem. Deciding whether $Q_{n}$ contains a hamiltonian cycle avoiding a given set of faulty edges is known to be NP-complete ([3]). Does a similar result hold for the variant with faulty vertices?

\section{References}

[1] R. CAHA, V. KOUBEK, Hamiltonian cycles and paths with a prescribed set of edges in hypercubes and dense sets, to appear in J. Graph Theory.

[2] R. Caha, V. Koubek, Spanning multi-paths in hypercubes, to appear in the Proceedings of Eurocomb '03 (European Conference on Combinatorics, Graph Theory and Applications, Prague, September 8-12, 2003).

[3] M. Y. CHAN, S-J. LEE, On the existence of Hamiltonian circuits in faulty hypercubes, SIAM J. Discrete Math. 4 (1991), 511-527.

[4] T. DvořÁK, Hamiltonian cycles with prescribed edges in hypercubes, to appear in SIAM J. Discrete Math.

[5] T. DVOřÁK, P. GREGOR, Hamiltonian paths with prescribed edges in hypercubes, submitted to the Proceedings of Eurocomb '03 (European Conference on Combinatorics, Graph Theory and Applications, Prague, September 8-12, 2003).

[6] J.-S. Fu, Fault-tolerant cycle embedding in the hypercube, Parallel Comput. 29 (2003), 821-832.

[7] F. Harary, Graph Theory. Addison-Wesley, 1969.

[8] F. HaRARY, J. P. Hayes AND H-J. WU, A survey of the theory of hypercube graphs, Comput. Math. Applic. 15 (1988), 277-289. 
[9] I. HAVEL, On hamiltonian circuits and spanning trees of hypercubes, Čas. Pěst. Mat. 109 (1984), $135-152$.

[10] M. Kobeissi, M. Mollard, Disjoint cycles and spanning graphs of hypercubes, Discrete Math. 288 (2004), 73-87.

[11] F. T. LeIghton, Introduction to Parallel Algorithms and Architectures: Arrays, Trees, Hypercubes. Morgan Kaufmann, San Mateo, CA 1992.

[12] M. LEWINTER, W. WIdUlski, Hyper-Hamilton laceable and caterpillar-spannable product graphs, Comput. Math. Appl. 34 (1997), 99-104.

[13] C. SAVAGE, A survey of combinatorial Gray codes, SIAM Rev. 39 (1997), 605-629.

[14] C.-H. TSAI, Linear array and ring embeddings in conditional faulty hypercubes, Theor. Comput. Sci. 314 (2004), 431-443. 
